\title{
Síndrome de Sweet - a propósito da localização incomum da forma clássica
}

\author{
Sweet Syndrome - the uncommon localization of the classical presentation
}

\author{
Heloísa Ribeiro ${ }^{1}$, Horácio Scigliano² ${ }^{2}$ Ermelinda Gonçalves ${ }^{1}$ \\ ${ }^{1}$ Serviço de Medicina Interna, Centro Hospitalar de Entre o Douro e Vouga, Santa Maria da Feira, Portugal \\ ${ }^{2}$ Lab. Anatomia Patológica Dr. Albino Oliveira, Centro Hospitalar entre Douro e Vouga, Santa Maria da Feira, Portugal
}

\begin{abstract}
Resumo
A Síndrome de Sweet é uma patologia incomum associada a diversas entidades. Descreve-se 0 caso de uma mulher de 60 anos com clínica de infeção respiratória, taquicardia de complexos estreitos e extensas lesões nos membros inferiores de aparecimento súbito, cuja histologia foi compatível com S. Sweet, tendo completado 4 semanas de corticoterapia, sem recorrência por mais de seis meses após o evento.
\end{abstract}

Palavras-chave: Síndrome de Sweet, Infeção Respiratória, lesões cutâneas

\section{Introdução}

A Síndrome de Sweet constitui uma patologia dermatológica incomum, que se associa ao aparecimento de lesões cutâneas dolorosas que atingem principalmente cabeça, braços e tronco. A sua causa não é conhecida, podendo estar associada a diversos fatores, como infeções, neoplasias e fármacos. A corticoterapia sistémica constitui o tratamento recomendado. ${ }^{1}$

\section{Caso Clínico}

Descreve-se o caso de uma doente do sexo feminino, de 60 anos de idade, autónoma nas atividades da vida diária. Apresentava antecedentes de Asma diagnosticada aos 14 anos, Hipertensão Arterial, Dislipidemia e intervenção cirúrgica a pólipos uterinos em data que não sabia especificar. Encontrava-se habitualmente medicada com Sinvastatina $20 \mathrm{mg} / \mathrm{dia}$, Losartan/Hidroclorotiazida $100+25 \mathrm{mg} / \mathrm{dia}$, Montelucaste $10 \mathrm{mg} / \mathrm{dia}$ e Fluticasona/salmeterol que efectuava em SOS.

Recorreu ao Serviço de Urgência por tosse irritativa e dispneia em agravamento progressivo com uma semana de evolução. No dia anterior à admissão descrevia o aparecimento de lesões na face interna das coxas, associada a sensação de frio no local, com aspeto circular, infracentimétricas, que não desapareciam à digi-

\begin{abstract}
Sweet Syndrome is an uncommon dermatological condition which can be associated with several different factors. We present a case of a 60 years old women with an upper respiratory infection, narrow complexes tachycardia and acute onset of extensive lower limbs skin lesions, which histology was compatible with Sweet Syndrome. She had been medicated with corticotherapy for 4 weeks, without recurrence more than 6 months later. Keywords: Sweet Syndrome, Upper respiratory infection, skin lesions
\end{abstract}

topressão e, na porção inferior de ambas as pernas, descritas com dimensões superiores (cerca de 3-4 cm), mais rarefeitas e com padrão de cor irregular. Sem febre, palpitações, dor torácica, sintomas urinários ou gastrointestinais. Ao exame objetivo apresentava-se vigil e orientada, ligeiramente descorada mas hidratada, apirética, com tensão arterial de 144/64 mmHg; pulso radial de 150 bpm, frequência respiratória de 24 cpm, com discreta tiragem supraclavicular, saturação de 92\% em ar ambiente. Auscultação cardíaca com taquicardia e pulmonar com sibilos bilaterais e crepitações na base direita. De realçar a presença de lesões petequiais e purpúricas nos membros inferiores, sem edema periférico (Figura 1).

Da investigação efectuada no Serviço de Urgência salienta-se gasimetria arterial com p02 $65 \mathrm{mmHg}$, sem outras alterações relevantes, electrocardiograma com taquicardia regular de complexos estreitos com FC 150 bpm tendo efetuado amiodarona com reavaliação em ritmo sinusal. Analiticamente salientava-se anemia normocítica e normocrómica com hemoglobina 11,7 g/ dL, 9x10^9/L leucócitos com 8, 3×10^9 neutrófilos, velocidade de sedimentação 102 mm, proteína C reativa 49,9 mg/L, hormona estimulante da tiróide 0,16 mUl/L com T4 livre normal e urina tipo II com 250 leucócitos, sem outras alterações. Raio X tórax com hiperinsuflação pulmonar, alterações fibróticas bilateralmente e infiltrado para hilar direito, tendo realizado tomografia axial compu-

Figura 1. Lesões cutâneas nas coxas e pernas na admissão Serviço de Medicina Interna

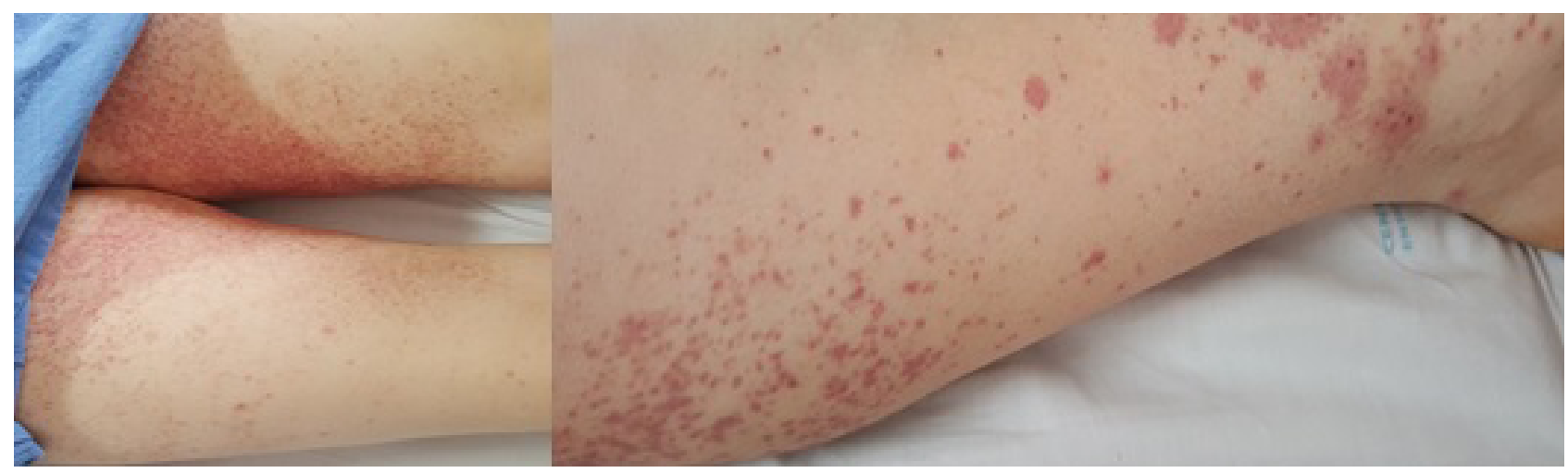


Figura 2. (A) Pele com pustulose confluente intraepidérmica, espongiose e imagens de leucocitoclastia na derme. (HE; 40x)

(B) Interfase dermoepidérmica. Observa-se foco de pustulose na área basal da epiderme e imagens de leucocitoclastia na derme (HE; 600x)

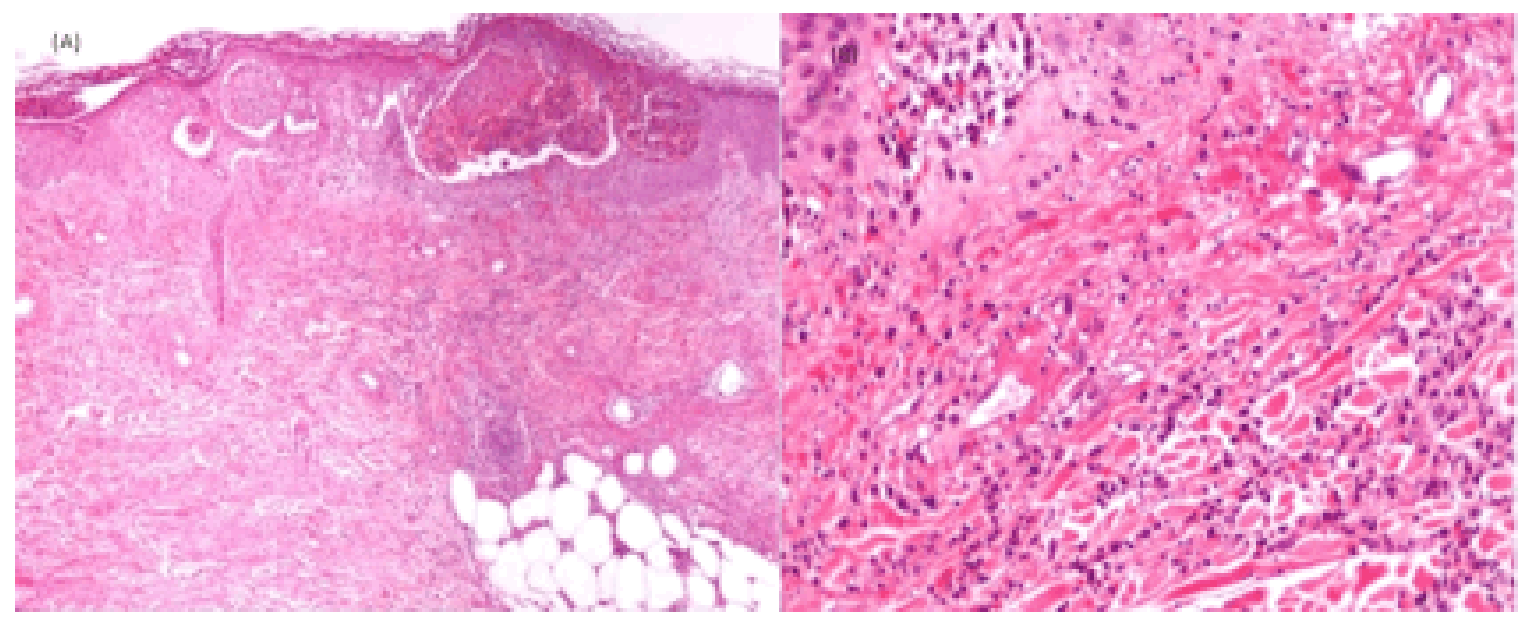

tadorizada onde se descreveu "Extensas lesões esclerofibróticas sequelares predominando em ambos os lobos inferiores e lobo médio, associando-se à direita, no vértice do lobo inferior, a opacidade mal definida, sugerindo processo inflamatório/infecioso em fase aguda".

A doente foi admitida no Serviço de Medicina Interna com diagnóstico de Pneumonia Adquirida na comunidade, tendo cumprido antibioterapia com amoxicilina e ácido clavulânico 1,2gr 8/8h (total de 7 dias) e azitromicina $500 \mathrm{mg} / \mathrm{dia}$ (durante 3 dias), corticoterapia (prenisolona $40 \mathrm{mg} / \mathrm{dia}$ ) e verapamilo (80 mg 8/8h) para controlo da frequência cardíaca. Tendo em conta as lesões cutâneas e alterações analíticas constatadas no estudo inicial, foi solicitado estudo complementar salientando-se cinética do ferro com capacidade de fixação ferro discretamente diminuída, sem défice de vitamina B12 ou ácido fólico; anticorpos IgG anti Mycoplasma pneumoniae reativo e anti Chamydophila pneumoniae positivo (42 UA/mL) com anticorpos IgM negativos para ambos os microorganismos; enzima de conversão da Angiotensina, calcémia e beta 2-microglobulina normais, electroforese de proteínas com aumento policlonal das imunoglobulinas; sorologias negativas para o vírus da imunodeficiência humana, hepatite B e C; sem consumo de complemento, anticorpo antinucleares e anti-citoplasma de neutrófilo negativos, fator reumatóide discretamente elevado com anticorpo anti-citrulina negativo. Ecocardiograma transtorácico com insuficiência mitral mínima, fluxo diastólico transmitral sugestivo de anomalia de relaxamento, sem outras alterações descritas.
Realizou biópsia de pele onde se descreveu imagens sugestivas de eritema reativo, de tipo síndrome de Sweet, sendo descrito nos cortes microscópicos examinados com técnica de Hematoxilina e Eosina (HE) epiderme com agregados pustulares intraepidérmicos, confluentes, associados a espongiose e apoptose focal de queratinócitos; a derme papilar revela-se edemaciada com imagens de leucocitoclastia; na derme profunda observam-se estruturas vasculares arteriolares ectásicas, revestidas de endotélio alto, de núcleos hipertróficos, envolvidas por infiltrado inflamatório de polimorfonucleares neutrófilos, com piócitos, que ainda atinge as glândulas sudoríparas e o tecido adiposo hipodérmico (Figura 2).

Neste contexto efectuou rastreio de neoplasias adequado à idade e escalação etário com tomografia axial abdomino-pélvica sem evidência de neoplasia, ecografia tiroideia sem alterações descritas, mamografia e ecografia mamária com padrão mamário com densidades fibroglandulares dispersas, sem alterações significativas, nomeadamente de características suspeitas, sem nódulos sólidos ou líquidos bilateralmente, sem alterações dos tegumentos ou adenomegalias axilares (classificação BI-RADS 1); colpocitologia em meio líquido sem achados neoplásicos e endoscopia digestiva alta e baixa sem alterações.

Após a alta hospitar a doente completou quatro semanas medicada com prednisolona $40 \mathrm{mg} / \mathrm{dia}$, tendo-se verificado resolução completa das lesões cutâneas (Figura 3), posteriormente com desmame completo de corticoterapia, sem recrudescimento após mais de 6 meses após suspensão da mesma. Analiticamente evoluiu

Figura 3. Lesões cutâneas após 2 semanas de corticoterapia

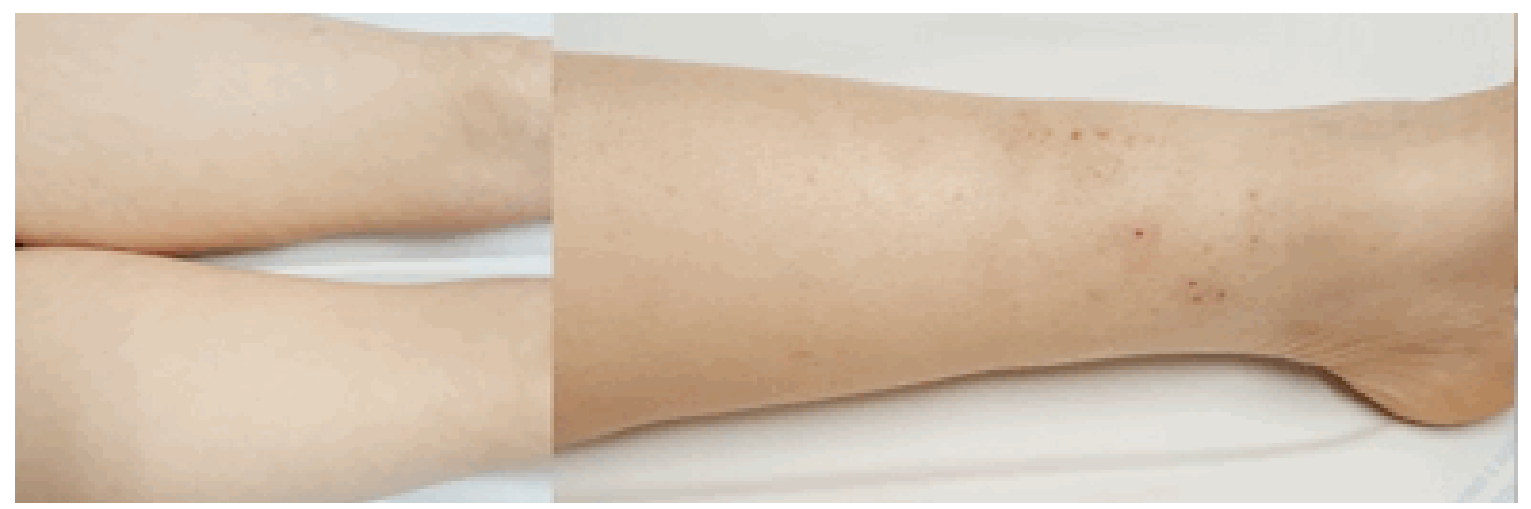


com correção da síndrome inflamatória sistémica, nomeadamente com normalização da hemoglobina, velocidade de sedimentação e proteína C-reativa. Foi constatada igualmente normalização da electroforese de proteínas.

\section{Discussão}

A pele pode apresentar alterações associadas a um vasto número de condições, de que é exemplo a toma de fármacos, presença de doenças infeciosas, neoplásicas, auto-imunes e endócrinas. ${ }^{1}$ No caso da doente apresentada a integração da história clínica e exame objetivo levou numa primeira fase à exclusão de vasculite e doenças infeciosas associadas a manifestações cutâneas, nomeadamente infeção por microorganismos atípicos e endocardite. A biópsia cutânea desempenhou um papel fundamental no diagnóstico e estudo subsequente da doente ao ser compatível com Síndrome de Sweet. ${ }^{2-4}$

A Síndrome de Sweet é descrita principalmente em três contextos: S. Sweet clássica ou idiopática, associada a neoplasia ou induzida por fármacos. ${ }^{5-8}$ Embora a forma clássica seja mais comum em mulheres e entre os 30 a 60 anos de idade, é mais frequente nos membros superiores (80\%), cabeça e pescoço (50\%) e tronco (30\%), sendo pouco frequente nos membros inferiores. Do ponto de vista analítico está descrita neutrofilia em $80 \%$ dos casos e elevação da velocidade de sedimentação em 90\%, sendo pouco frequente a presença de anemia. ${ }^{5}$ Apesar de, de acordo com os critérios de diagnóstico revistos por von den Driesch em 1994 (Tabela 1), ${ }^{5,6}$ a doente apresentar dois critérios major (aparecimento súbito de lesões cutâneas que podem ser placas eritematosas dolorosas ou nódulos e achados histopatológicos típicos) e três critérios minor (infeção respiratória prévia, excelente resposta à corticoterapia e analiticamente, na apresentação, velocidade de sedimentação eritrocitária e proteína $C$ reativa aumentadas, contagem de glóbulos brancos $>8 \times 10^{9} / \mathrm{L}$ com $>70 \%$ de neutrofilia), dada a localização nos membros

Tabela 1. Critérios de diagnóstico de Síndrome de Sweet clássica

\begin{tabular}{|c|}
\hline Major \\
\hline $\begin{array}{c}\text { 1) Início súbito de placas ou nódulos eritematosos dolorosos; } \\
\text { 2) Evidência histopatológica de infiltrado neutrofilico denso sem } \\
\text { evidência de vasculite leucocitoclástica; }\end{array}$ \\
Minor \\
1) Pirexia >38; \\
2) Associação com doença inflamatória, hematológica, neoplasia ou \\
gravidez ou precedida por infecção respiratória, gastrointestinal ou \\
vacinação; \\
3) Excelente resposta a corticoterapia sistémica ou iodeto de \\
potássio; \\
4) Alterações analíticas a apresentação (3 de 4): velocidade de \\
sedimentação $>20$ mm/h, Proteína C reativa elevada, contagem de \\
glóbulos brancos $>8 \times 10^{9}$, > 70\% de neutrófilos da contagem total \\
de glóbulos brancos;
\end{tabular}

inferiores e presença de anemia, achados mais frequentes na síndrome associada a neoplasias (do foro hematológico e tumores sólidos), foi necessário progredir estudo de modo a despistar presença concomitante de neoplasias, tendo sido já previamente descartada pela história clínica fármacos potencialmente associados.

Embora na forma clássica as lesões possam resolver espontaneamente, o tratamento de primeira linha é composto por prednisolona $1 \mathrm{mg} / \mathrm{Kg} / \mathrm{dia}$ (usualmente 30 a $60 \mathrm{mg} /$ dia) a efetuar desmame em 4 a 6 semanas (embora alguns doentes possam necessitar de ciclos mais prolongados), colchicina e iodeto de potássio, enquanto o tratamento de segunda linha é constituído por fármacos como a indometacina e ciclosporina, podendo ser necessário o recurso a antibioterapia na presença de sobreinfeção bacteriana. ${ }^{5,6,9 \text {, }}$ 10 Pode observar-se recorrência da síndrome após remissão por períodos variáveis de forma espontânea ou induzida por tratamento. 8,10

\section{Conclusão}

A Síndrome de Sweet implica o diagnóstico diferencial com outras entidades, nomeadamente outras patologias sistémicas e o estudo aprofundado para exclusão de tumor sólido e neoplasia hematológica, principalmente tendo em conta a localização das lesões, pouco frequente na forma clássica.

\section{Bibliografia}

1. Cahill J, Sinclair R. Cutaneous manifestations of systemic disease. Aust Fam Physician. 2005; 34(5): 335-340.

2. Leitão J, Reis C, Malcata A, Carvalho A, Feio M, Perdigoto R, et al. Síndrome de Sweet, Afecção cutânea benigna ou sistémica grave? Medicina Interna. 1996; 3: 24-28.

3. Callen JP. Neutrophilic dermatoses. Dermatol Clin. 2002; 20(3): 409-19.

4. Malone JC, Slone SP, Wills-Frank LA, Fearneyhough PK, Lear SC, Goldsmith LJ, et al. Vascular inflammation (vasculitis) in Sweet syndrome: a clinicopathologic study of 28 biopsy specimens from 21 patients. Arch Dermatol 2002; 138: 345-349.

5. Cohen PR. Sweet's syndrome - a comprehensive review of an acute febrile neutrophilic dermatosis. Orphanet J Rare Dis. 2007; 2: 34.

6. Raza S, Kirkland RS, Patel AA, Shortridge JR, Freter C. Insight into Sweet's syndrome and associated-malignancy: A review of the current literature. Int J Oncol. 2013; 42(5): 1516-1522.

7. Bonamigo RR, OIm GS, Razera F. Dermatoses neutrofilicas - Parte I. An Bras Dermatol. 2011; 86(parte 1): 11-27.

8. Lima-Wojcik AS, Nishimori FS, Santamaria JR. Síndrome de Sweet: estudo de 23 casos. An Bras Dermatol. 2011; 86(2): 265-271.

9. Cohen PR. Neutrophilic dermatoses: a review of current treatment options. Am J Clin Dermatol. 2009; 10(5): 301-12.

10. Cohen PR, Kurzrock R. Sweet's syndrome revisited: a review of disease concepts. Int J Dermatol. 2003; 42: 761-78. 